\title{
Research on Small Hydropower Generation Forecasting Method Based on Improved BP Neural Network
}

\author{
LI Miao ${ }^{1, a}$, DENG Chang Hong ${ }^{1, b}$, TAN Jin ${ }^{1, c}$, YANG Wei ${ }^{1, d}$ ZHENG Li $i^{2, e}$ \\ ${ }^{1}$ WuHan, HuBei Province, China \\ ${ }^{2}$ LongQuan, ZheJiang Province, China \\ alimiao227@126.com, bdengch@whu.edu.cn, \\ 'tanjin93@163.com, ${ }^{\mathrm{d}} 476824797 @ q q . c o m,{ }^{\mathrm{e}} 569397094 @ q q . c o m$
}

Keywords: Correlation Analysis;Small hydropower forecast; BP network; Wavelet Decomposition

\begin{abstract}
Small hydro-power generation shows strong uncertainty, which greatly affects the load forecasting work in small hydropower regions. Thus it's important to improve the accuracy of small hydropower generation load forecasting. At present, the most commonly used forecasting method is artificial neural network, which has strong adaptability and learning ability but poor generalization and easily falls into local minimum. The random fluctuation of small hydropower is not taken into consideration. This paper was based on analyzing the characteristics of small hydropower generation load, combining the wavelet transform to decompose the historical load to establish the prediction model for each component feature. Particle Swarm Optimization (Algorithm) was used to optimize initial weights and thresholds of neural networks before the prediction. After verified by real case in a rich small hydropower area in some province, the load prediction precision reaches $93.7 \%$, higher than the precision of the high-voltage system criteria for assessing. The accuracy and effectiveness of the method is verified.
\end{abstract}

\section{Introduction}

The large scale access and locally consumption of DG in distribution network has made the traditional distribution network gradually transform into $\mathrm{AND}^{[1]}$. DG mainly includes solar photovoltaic power generation, wind power generation, small hydropower, etc. The characteristics of no pollution and quick effect make DG play an important role in energy conservation and emission reduction. But the large scale access of DG made the traditional load forecasting method no longer applicable ${ }^{[2]}$. Taking the distribution network with high permeability of small hydropower for an example, the uncertainty of small hydropower generation load leads to the poor regularity of network load. The traditional load forecasting method has low accuracy. Thus, research on load forecasting method for small hydropower generation is of great significance for ADN ${ }^{[3 \sim 4]}$.

Most load forecasting methods at present are aimed at traditional load, but few taking the influence of small hydropower into consideration. The paper [5] proposed two stage reduction method but it did not set up an effective forecasting model aiming at the uncertainty of small hydropower. The paper [6] proposed a new combined forecasting method for small hydropower generation but the process of establishing wavelet regression combined forecasting model in this method is comparatively complex and difficult to realise. The paper [7] established the regression relationship between meteorological factors and meteorological load, but it did not take accumulated rainfall into consideration and ignored the cumulative effect of small hydropower.

This paper analyses the characteristics of daily load curve and time series characteristics of small hydropower. Then decompose the original load using wavelet decomposition method and select the corresponding input variables for the prediction model based on the characteristics each component after decomposition. BP neural network algorithm optimized by PSO is used for each component prediction model and finally obtain the prediction results after the reconstruction of the predictive value of each component. BP neural network has strong learning ability, but it is easy to fall into local 
optimum $^{[8]}$. Thus, this paper utilize PSO algorithm with global optimization ability to optimize the initial weights and thresholds of the neural network. This method is used to analyze the network load of some province rich in small hydropower. The prediction accuracy is significantly improved and the validity and superiority of this method are verified.

\section{Analysis of small hydropower generation characteristics}

Small hydropower generation load has different regularity compared to general power load and the characteristic analysis of small hydropower generation is necessary.

Characteristics of small hydropower load curve. The small hydropower generation load curve shows the properties of generation load intuitively. Figure 1 is the small hydropower generation load curve for two weeks. Because of the specific properties of small hydropower generation, the performance affected by the feed-in tariff should be considered in establishing a prediction model.

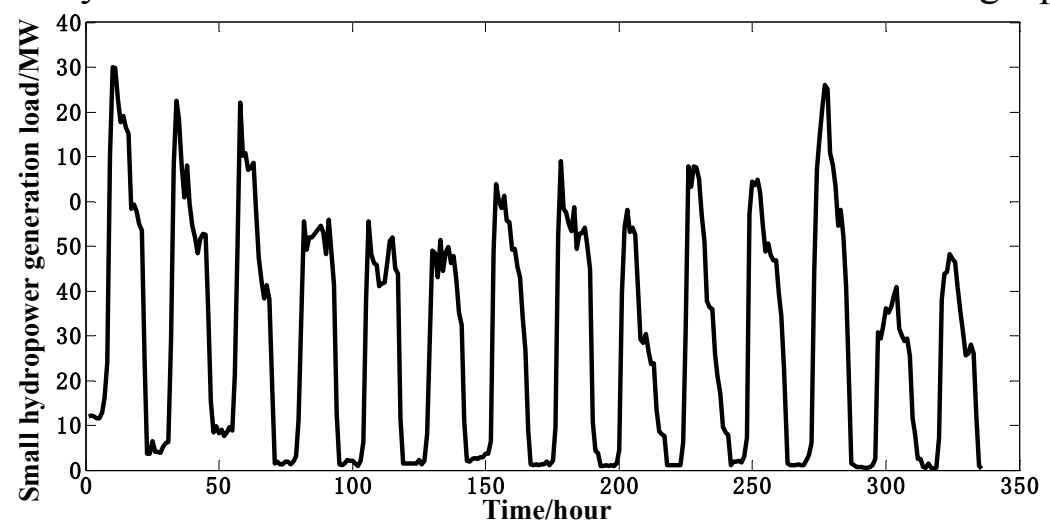

Fig. 1. Small hydropower generation load curve of continuous two weeks

Characteristics of small hydropower load time series. The small hydropower load is a typical time series. Research on self correlation of load time series helps to understand the characteristics of itself and establish a more efficient load forecasting model.

Assume there is $L$ days' historical data and $X_{i}$ is set to be load column of $i$, which includes 24 points load value. $X_{i}(i=1,2, \ldots, L)$ is stored according to the principle of near the date to form the matrix $X_{\text {parallel }}$ written as:

$$
X_{\text {parallel }}=\left[X_{1}, X_{2}, \ldots, X_{L}\right]_{24 \times L}
$$

The correlation coefficient of any two columns of $X_{\text {parallel }}$ is set as:

$$
R_{i, j}=\frac{E\left\{\left(X_{i}-E(X)\right)\left(X_{j}-E(X)\right)\right\}}{\sqrt{E\left\{\left[X_{i}-E(X)\right]^{2}\right\}} \sqrt{E\left\{\left[X_{j}-E(X)\right]^{2}\right\}}}
$$

$E$ is the average symbol and the daily load curve correlation coefficient matrix is defined as:

$$
\left\{\begin{array}{cc}
B=\left[b_{i j}\right]_{(L-7) \times 7} & (i=1,2, \ldots, L-7) \\
b_{i j}=R_{i, i+j} & (j=1,2, \ldots, 7)
\end{array}\right.
$$

The line $i$ of the matrix shows the correlation coefficient of the day $i$ and 1 to 7 day before. By averaging the columns in matrix $B$, the average correlation coefficient $S_{i}$ of each column is obtained.

$$
S_{j}=\frac{\sum_{i=1}^{L-7} b_{i j}}{L-7}(i=1,2, \ldots, 7)
$$

$S_{1}$ reflects the daily periodicity of the load, and $S_{7}$ reflects weekly periodicity of the load. Calculated by the above method, the correlation coefficient of daily load curve with the former 1 to 7 day is compared in Figure 2. 


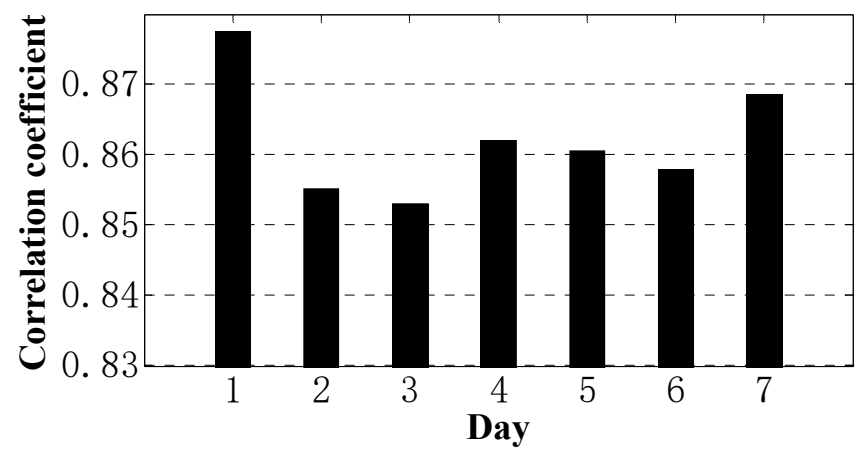

Fig. 2 Comparison figure of the correlation coefficient between

By using the same calculation method, the vertical correlation of small hydropower generation load is obtained and the calculation results are shown in Figure 3.

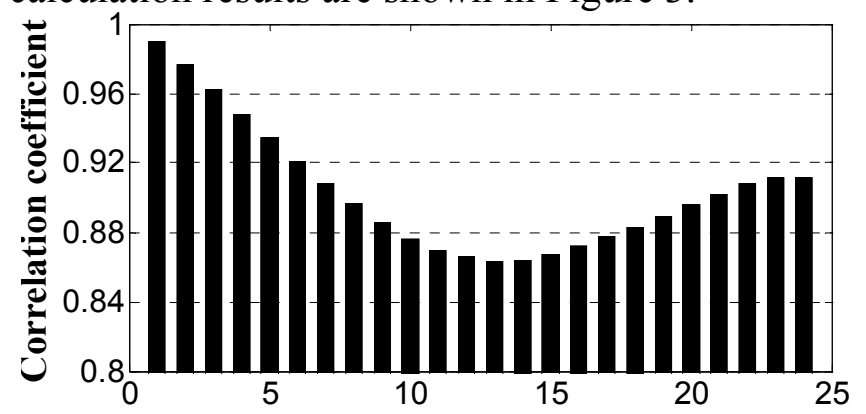

Time/hour

Fig. 3 Calculation results of vertical correlation of small hydropower load

Known from the above analysis of the characteristics and time series characteristics of the small hydro power load curve, the daily rainfall and the accumulated rainfall before the forecast days should be considered as the important input variables of the forecasting model.

Small hydropower generation load forecasting method

Select forecast model input variables. This paper uses the wavelet transform to decompose the historical load sequence of small hydropower. Discrete wavelet transform is used to decompose and reconstruct it as follows:

$$
W_{f}(j, k)=\frac{1}{\sqrt{a_{0}^{j}}} \int_{R} f(x) \bar{\varphi}\left(a_{0}^{-j} x-k b_{0}\right) d x
$$

$a$ and $b$ represent the dilation factor and displacement factor of $\varphi_{a, b}(t)$, respectively. $\bar{\varphi}(t)$ is the conjugate complex number of $\varphi(t) \cdot \bar{\varphi}(t)$ is the mother wavelet. The original small hydropower load is decomposed to three scales as figure $4 . Y$ is the original sequence. $M$ is the low frequency component of low-pass filter. $N$ is the high frequency of high-pass filter.

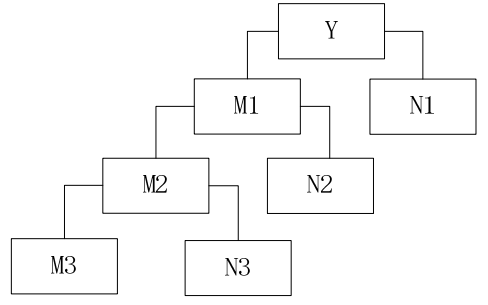

Fig. 4 Wavelet three scale decomposition model

Figure 4 shows that the multi-resolution analysis of each layer is the only decomposition of low frequency component. The decomposition formula is showed as follows:

$$
Y=M 3+N 1+N 2+N 3
$$

In the original load series, load with slower varying frequency is the low frequency component. Small hydropower historical data is decomposed to three scale wavelet by using db2 decomposition. The diagram of each load component after $\mathrm{db} 2$ decomposition is as follows: 

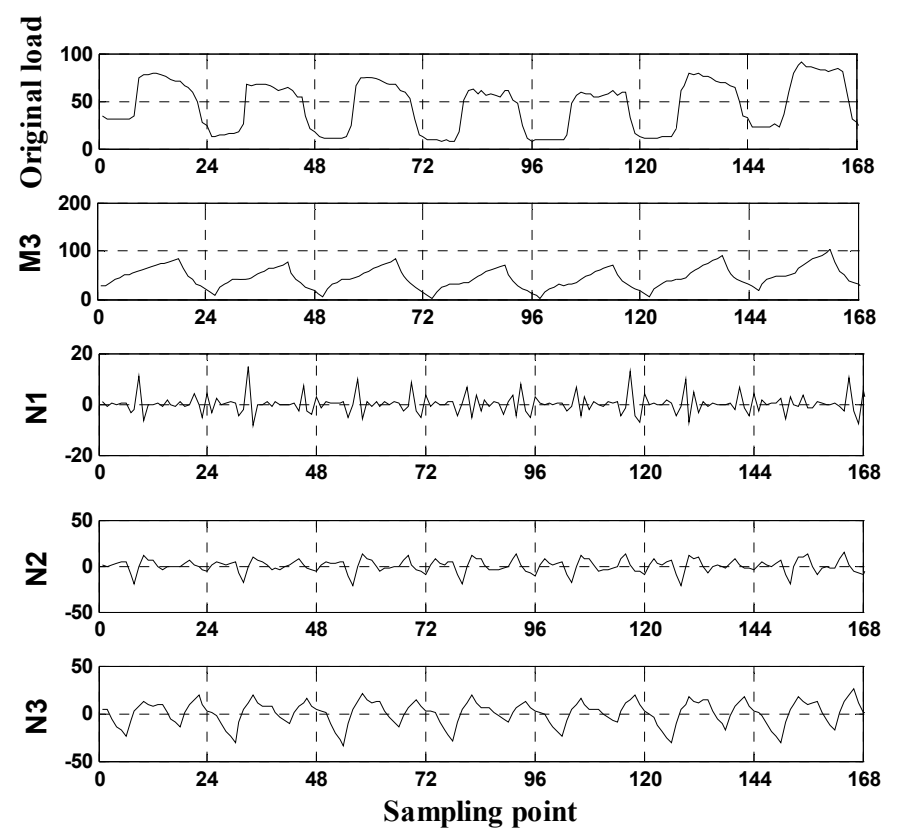

Fig. 5 Each load component after decomposition

Figure 5 show that M3 has obvious daily periodicity. N1 and N2 have certain daily periodicity and randomness, while N3 has both moment periodicity and daily periodicity. This paper selects the input variables of prediction model as showed in table 1 .

Tab. 1 Input variables component prediction model

\begin{tabular}{|c|c|}
\hline component & Input variables \\
\hline M3 & 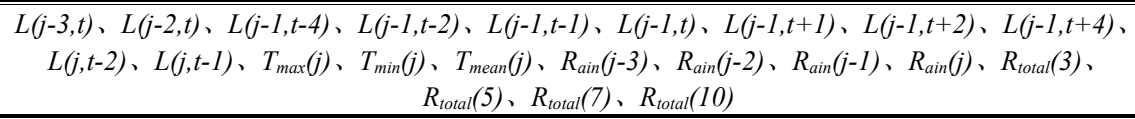 \\
\hline N3 & 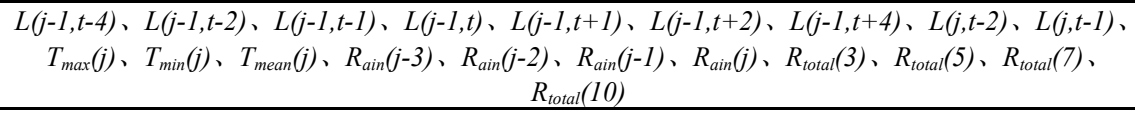 \\
\hline N1、N2 & $\begin{array}{c}L(j-1, t-2), L(j-1, t-1), L(j-1, t), L(j-1, t+1), L(j-1, t+2), L(j, t-2), L(j, t-1), T_{\max }(j), T_{\min }(j), \\
T_{\text {mean }}(j) 、 R_{\text {ain }}(j-3), R_{\text {ain }}(j-2), R_{\text {ain }}(j-1) 、 R_{\text {ain }}(j), R_{\text {total }}(3), R_{\text {total }}(5), R_{\text {total }}(7), R_{\text {total }}(10) \\
\end{array}$ \\
\hline
\end{tabular}

In the table, $R_{\text {total(3) }} 、 R_{\text {total(5) }} 、 R_{\text {total(7) }} R_{\text {total(10) }}$ represent the cumulative rainfall of 3,5,7,10 days before the forecast data, respectively. $R_{\text {ain }(j)}$ represents the rainfall of day $j . T_{\operatorname{mean}(j)} 、 T_{\min (j)} 、 T_{\max (j)}$ represent the average temperature, maximum temperature, minimum temperature of day $j$, respectively. $L_{(j, t)}$ represents the load of time $t$, day $j$.

Modified BP neural network prediction model . The working state of BP neural network model is stable, but it has shortcomings such as easy to fall into local minimum point. In order to avoid the network converging to a local minimum value, this paper uses PSO $\mathrm{w}$ to optimize the BP neural network's initial weights and threshold.

Assuming there is a D-dimensional target search space and a group made up of $\mathrm{m}$ particles, $X_{i}=\left(x_{i}{ }^{1}, x_{i}{ }^{2}, \ldots, x_{i}{ }^{D}\right)$ represents the position of particle $i(i=1,2, \ldots, m) . V_{i}=\left(v_{i}{ }^{l}, v_{i}{ }^{2}, \ldots, v_{i}{ }^{D}\right)$ is the velocity of particle $i . P_{g}=\left(p_{g}{ }^{l}, p_{g}{ }^{2}, \ldots, p_{g}{ }^{D}\right)$ is the best location that all particles have experienced. $P_{i}=\left(p_{i}{ }^{l}, p_{i}{ }^{2}, \ldots, p_{i}{ }^{D}\right)$ is the best location that individual particle has experienced. Particle swarm algorithm updates the position of particles through the formula 7 and $8 . r_{1}$ And $r_{2}$ are the random numbers transforming in the $[0,1] . c_{1}$ and $c_{2}$ are acceleration constant. $\omega$ is inertial factor. $\alpha$ is constant factor.

$$
\begin{gathered}
v_{i}^{d}=\omega v_{i}^{d}+c_{1} r_{1}\left(p_{i}^{d}-x_{i}^{d}\right)+c_{2} r_{2}\left(p_{g}^{d}-x_{i}^{d}\right) \\
x_{i}^{d}=x_{i}^{d}+\alpha v_{i}^{d}
\end{gathered}
$$

Example application

This paper takes an area with high permeability of small hydropower as the research object. According to table 1, it builds the forecasting model and use BP neural network algorithm improved 
by PSO to forecast the load of each component, respectively. Forecasting results are shown in figure 6.
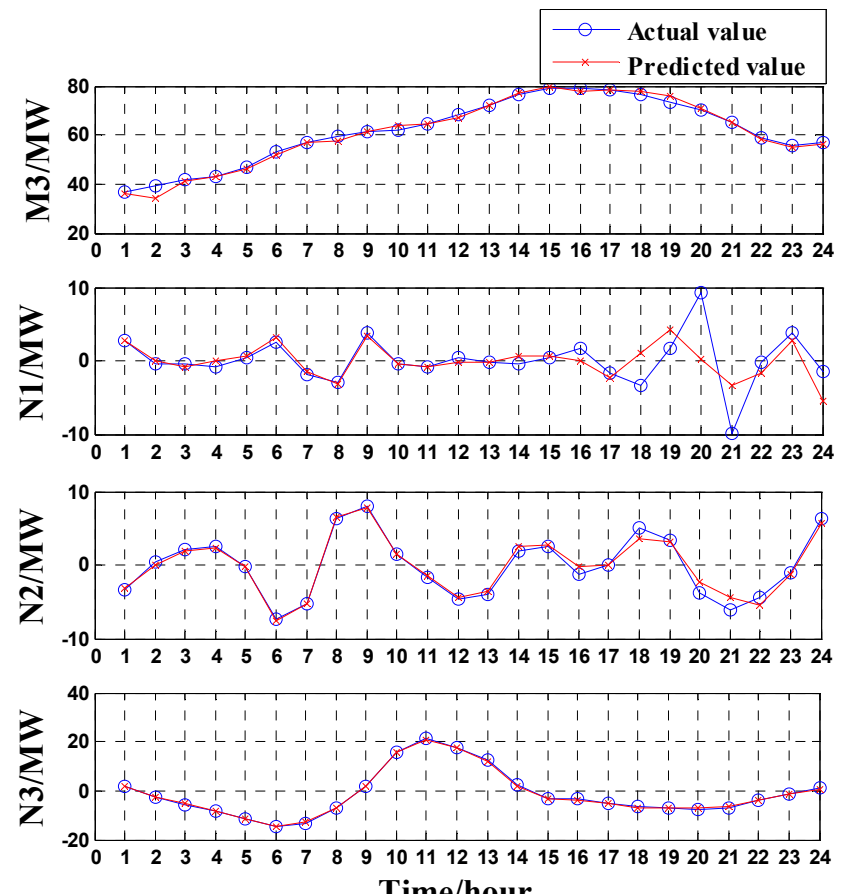

Time/hour

Fig.6 Forecasting results of each load component

The predictive value 1 is obtained by doing the combination of each load component predictive value as shown in figure 7 . The predictive value 2 is obtained by the forecasting method without wavelet decomposition. Figure 7 shows that the prediction effect after wavelet decomposition and synthetic is much better than that of using original data directly.

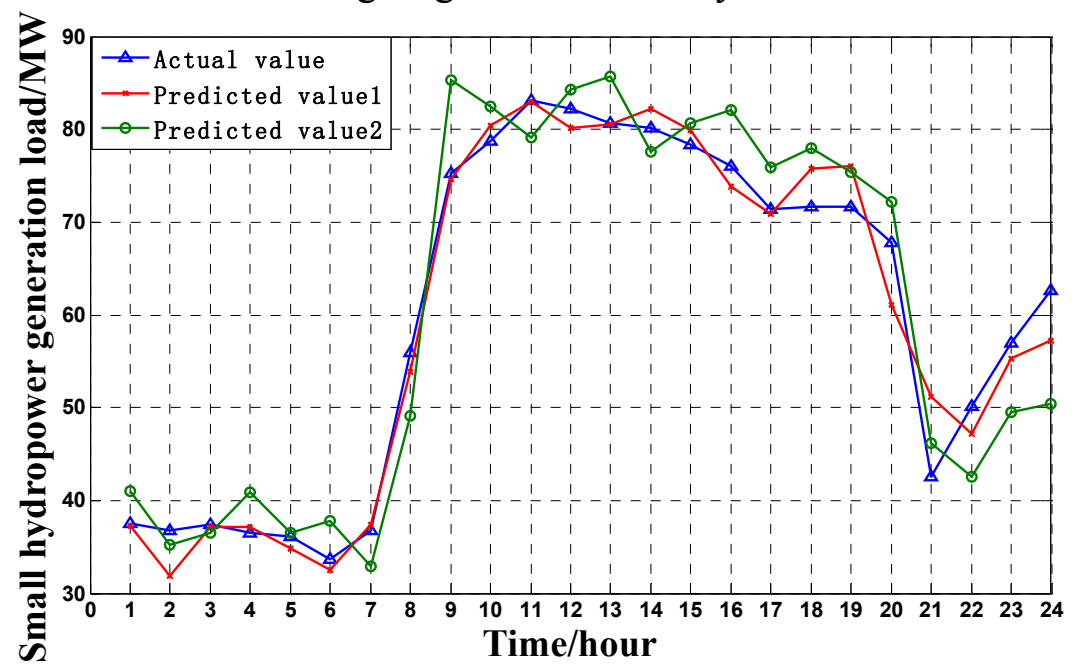

Fig.7 Small hydropower generation load forecasting results

The computation formula of load forecasting deviation rate $E_{i}(\%)$ is as follows:

$E_{i}=($ forecasting value- actual value $) /$ actual value $\times 100 \%$

The computation formula of daily load forecasting accuracy $A_{L}(\%)$ is as follows:

$$
\mathrm{A}_{L}=\left[1-\sqrt{\frac{1}{n} \sum_{i=1}^{n} E_{i}^{2}}\right] \times 100 \%
$$

This paper calculates 24 points prediction accuracy through formula (9). The prediction accuracy of short-term load forecasting method which uses the improved BP neural network based on swarm wavelet in this paper reached $90.6 \%$ and it is higher than the general prediction method. Furthermore, the prediction accuracy was up to $93.7 \%$ by combining swarm wavelet and improved BP neural network. 


\section{Conclusion}

Taking the distribution network with high permeability of small hydropower as the research object, this paper proposed a load forecasting method for the present distribution network aiming at the strong uncertainty of DG. This paper analyzes the characteristics of small hydropower generation from two aspects of load curve characteristics and time series characteristics. Taking the advantages of wavelet transform to extract the characteristic information of the load sequence quickly, the primary small hydropower load is decomposed to build forecasting model respectively aiming at its characteristics of each component. In addition, taking into account that the BP neural network is easy to fall into local best characteristics, it was optimized through PSO algorithm and then reconstruct to small hydropower load forecast value. Finally it obtained the network value by combining the social electricity load and small hydropower load. Through the example validation, the load forecasting method proposed in this paper has higher accuracy and is of reference for improving the load forecasting accuracy of large scale DG access distribution network.

\section{Acknowledgements}

This work was financially supported by the National Science and technology support program (2013BAA02B00).

\section{References:}

[1] Zhang Jianhua, Zeng Bo, Zhang Yuying. Key issues and research prospects of active distribution network planning[J]. Transactions of China Electro-technical Society,29(2),pp:13-23,2014

[2] KANG Longyun, GUO Hongxia, WU Jie. Characteristics of distributed generation system and related research issues caused by connecting it to power system[J]. Power System Technology,34(11),pp:43-47.

[3] LI Shushan, LIAO Shengli, LI Gang. Integrated power generation scheduling compilation method for large-scale small hydropower station group $[\mathrm{J}]$. Proceedings of the Chinese Society for Electrical Engineering, 32(13), pp:39-35,2012.

[4] Anuradha Wijesinghe,Loi Lei Lai.Small hydro power plant analysis and development[C]//Electric Utility Deregulation and Restructuring and Power Technologies. Wei hai, Shandong, 2011:25-30.

[5] XU Wei, LUO Xin, LIU Mei. Application of two-phase reduction method in load forecasting for regions with abundant small hydropower[J]. Power System Technology, 33(8), pp:87-92,2009.

[6] QUAN Ge, HUANG Min-xiang, ZHOU Miao-fei. Application of new combination forecasting method in daily generation forecasting for regions with abundant small hydropower[J]. Energy Engineering.04, pp:20-26,2011.

[7] JIN Yi-xiong, DUAN Jian-min, YANG Jun-qiang. Weather line regression and combination load forecast of mountainous area contain small hydro-power unit[J].Relay, 35(14),pp:54-58,2007.

[8] PENG Xin-song, HE Hui, YAO Jiangang. Method of bus-load forecasting using BP neural network optimized by PSO $[\mathrm{J}]$. Proceedings of the Chinese Society of Universities, 22(5),pp:146-151,2010. 